\title{
Current available therapies and future directions in the treatment of malignant gliomas
}

\author{
Annick Desjardins ${ }^{1,2}$ \\ David A Reardon ${ }^{1,3}$ \\ James J Vredenburgh ${ }^{1,2}$ \\ 'The Preston Robert Tisch Brain \\ Tumor Center; ${ }^{2}$ Department of \\ Medicine; ${ }^{3}$ Department of Surgery, \\ Duke University Medical Center, \\ Durham, NC, USA
}

\begin{abstract}
The prognosis of patients diagnosed with malignant glioma (MG) remains poor. However, recent advances in neuro-oncology allowing a better understanding of this particular disease have allowed the development of new therapeutics. Many molecular genetic and signal transduction pathway targets have been identified that are now being investigated. Novel locoregional treatments, as well as strategies to improve regional delivery, are being evaluated. Studies of combinations of these approaches are also underway. In this review, we will discuss the current and future therapies under evaluation for the treatment of malignant gliomas.
\end{abstract}

Keywords: glioma, glioblastoma, targeted therapy, kinase inhibitor

\section{Introduction}

Primary central nervous system (CNS) tumors represent only $1.35 \%$ of all cancers and $2.2 \%$ of all cancer-related deaths. ${ }^{1}$ Unfortunately, the prognosis of the most frequent primary CNS tumors, malignant glioma $(\mathrm{MG})$, remains poor. Glial neoplasms represent about $40 \%$ of all primary CNS tumors, over three quarters being malignant. ${ }^{2} \mathrm{MG}$ include Word Health Organization (WHO) grade III: anaplastic astrocytoma (AA), anaplastic oligodendroglioma (AO), and anaplastic oligoastrocytoma (AOA); and WHO grade IV: glioblastoma multiforme (GBM), and gliosarcoma., ${ }^{3,4}$ Despite the current standard treatments for MG including surgical resection, radiation therapy, and chemotherapy, the survival of patients with MG remains dismal, with a median survival of 2 to 3 years for patients with AA and 9 to 12 months for GBM patients. ${ }^{5}$ Favorable prognostic factors including youth, absent or minimal neurological signs, complete surgical resection, and good performance status have been identified, but unfortunately, clinical recurrence or progression is nearly universal. For patients with disease recurrence/progression, available systemic chemotherapies offer modest clinical benefit with a 6-month progression-free survival (PFS) of less than $15 \%$ for GBM and $31 \%$ for AA, ${ }^{6}$ and a median overall survival (OS) of 25 weeks and 47 weeks for recurrent GBM and AA, respectively. ${ }^{6}$ In addition to the fatal prognosis, MG affects many patients in their forties and fifties, frequently terminating promising lives prematurely and depriving young families of parents and spouses. Clearly, more effective therapies are desperately needed for patients afflicted with these tumors.

\section{Temozolomide}

Radiation therapy (RT) has been the standard-of-care for MG until recently, while systemic chemotherapy has had a limited role. ${ }^{7}$ However, in a recent phase III study, Stupp et al reported results that made temozolomide (TMZ) (Temodar ${ }^{\circledR}$, Temodal $^{\circledR}$, Schering-Plough Corporation), a DNA methylator and a second-generation imidazotetrazine derivative, a standard adjuvant chemotherapy for GBM at first diagnosis. Patients were randomized into 2 groups. One group received concurrent RT and daily TMZ at $75 \mathrm{mg} / \mathrm{m}^{2}$, followed by 6 monthly cycles of TMZ $\left(150-200 \mathrm{mg} / \mathrm{m}^{2}\right.$ 
orally daily, days 1 through 5, every 28 days) and the control group received RT alone. Patients in the RT alone group were allowed to receive TMZ at the time of disease progression. A median survival of 14.6 months was observed in patients treated with concurrent RT and TMZ followed by 6 monthly cycles of TMZ, compared to 12.1 months for patients treated with RT alone. Also, the 2-year survival rate improved from $10.4 \%$ for RT alone to $26.5 \%$ in the RT-TMZ group. ${ }^{8}$ They observed no grade 3 or 4 hematologic toxicities in the RT alone group. In the RT-TMZ group, 12 patients (4\%) experienced grade 3 or 4 neutropenia and 9 patients $(3 \%)$ had grade 3 or 4 thrombocytopenia. During the adjuvant TMZ therapy of the RT-TMZ group, $14 \%$ of patients had any grade 3 or 4 hematologic toxicities (4\% grade 3 or 4 neutropenia, $11 \%$ grade 3 or 4 thrombocytopenia). During the RT period, severe infections occurred in 6 patients $(2 \%)$ in the RT alone group and in nine patients (3\%) in the RT-TMZ group, during adjuvant TMZ therapy, 12 patients (5\%) experienced severe infections. Other common non-hematologic toxicities included: moderate to severe fatigue (26\% RT alone group, 33\% RT-TMZ group), thromboembolic events (6\% RT alone group, 4\% RT-TMZ), pneumonia (2\% RT alone group, 1\% RT-TMZ group), and opportunistic infections (one patient in each group). Finally, 2 patients in the RT-TMZ group died of cerebral hemorrhage in the absence of a coagulation disorder or thrombocytopenia.

Gene methylation is an important cellular mechanism of transcription suppression. $\mathrm{O}^{6}$-methylguanine-DNAmethyltransferase (MGMT) is a critical DNA repair protein, which removes chloroethylation, or methylation damage from the $\mathrm{O}^{6}$ position of DNA guanines, protecting the tumor cells against alkylating and methylating chemotherapeutic agents. ${ }^{9}$ The presence of MGMT gene methylation predicts for lack of MGMT expression and subsequent TMZ sensitivity, whereas the absence of MGMT gene methylation (unmethylated MGMT) predicts MGMT expression and potential TMZ resistance. Analyses of tumor specimens from patients treated on the Stupp trial ${ }^{8}$ were performed to determine the methylation status of MGMT and its correlation with survival. Patients with MGMT gene methylation who received RT and TMZ achieved a median PFS of 10.3 months and a 2-year survival rate of $46 \%$, compared to 5.9 months median PFS and $22.7 \%$ 2-year survival rate for the patients having MGMT gene methylation but treated with RT alone. In comparison, patients with unmethylated MGMT achieved a median PFS of 5.3 months and a 2-year survival rate of $14 \%$ for the RT-TMZ group and 4.4 months and $<2 \%$, respectively for the group receiving only RT. ${ }^{10}$
It was concluded that patients with newly diagnosed GBM and MGMT gene methylation benefited the most from the addition of TMZ to RT. ${ }^{10}$

A possible approach to improve the survival of patients with unmethylated MGMT is molecules that can reverse the process, such as $\mathrm{O}^{6}$-benzylguanine (O6-BG), an $\mathrm{O}^{6}$-alkylguanine-DNA alkyltransferase inhibitor. Phase I studies of O6-BG with BCNU and TMZ have been limited by hematologic toxicities preventing therapeutic dose escalation of the chemotherapy. ${ }^{11,12}$

Even though the addition of TMZ to RT provides an important step forward in the overall treatment of this disease and nitrosoureas remain an option, more effective therapies are necessary. Several innovative treatment strategies, including targeted therapeutics as well as locally administered agents, are being studied.

\section{Targeted therapies}

The recent success of small-molecule inhibitors of signal transduction pathways in other cancers has propelled rapid development of similar therapies in the treatment of patients with MG. Cellular processes contributing to normal homeostasis, once disrupted, can contribute to malignancy and this by the presence of common molecular alterations in the signal transduction pathways, a communication network of regulatory molecules within the cell. Several growth factors, hormones, and cytokines regulate these cellular processes. Epidermal growth factor (EGF), platelet-derived growth factor (PDGF), vascular endothelial growth factor (VEGF), hepatocyte growth factor/scatter factor (HGF/SF), and insulin-like growth factor (IGF) are some of the relevant growth factor pathways in gliomas. Tyrosine kinases are associated with the receptors for these pathways. After ligand binding, the receptors undergo dimerization that permits transphosphorylation, in which the kinase domain of one receptor phosphorylates one or more intracellular tyrosine residues on the second receptor. Phosphotyrosine residues recruit adaptor proteins, activating downstream effector molecules that initiate signaling cascades to regulate gene transcription in the nucleus. MG are known to present overexpression or mutations of receptors and intracellular downstream effectors, leading to activation of signaling pathways, resulting in uncontrolled cellular proliferation, survival, and invasion. Several approaches are available to inhibit signaling pathways, from the inhibition of upstream growth factor ligands and their receptors, to the inhibition of downstream intracellular effectors. Specific inhibitors of these targets have shown promise in preclinical and clinical trials; however, significant work remains to maximize the 
utility of these treatments to improve patient outcomes. The major challenges in the use of kinase inhibitors facing the neuro-oncology community are: (1) identification of the optimal therapeutic target(s), (2) establishment of biomarkers of tumor sensitivity or resistance, and (3) optimization of signaling inhibitor combinations with either other inhibitors or cytotoxic agents for efficacy and toxicity, as unusual adverse events such as neurotoxicity, hypertension and cardiac events can occur from those combinations.

\section{Vascular endothelial growth factor}

Vascular proliferation, or neoangiogenesis, is a histopathological characteristic of MG. ${ }^{13,14}$ One attractive therapeutic target for many neoplasms is VEGF, the principal mediator of tumor angiogenesis. MG overexpresses VEGF, the levels of which correlate directly with tumor vascularity and grade, and inversely with prognosis. ${ }^{15-18}$ Tumor-associated endothelial cells express VEGFR2, creating a paracrine loop of angiogenic activation, indicating that VEGF and its receptors are important therapeutic targets. ${ }^{17,19}$

Bevacizumab (BV) is a humanized murine monoclonal antibody, binding VEGF-A ${ }^{20,21}$ and preventing interaction and activation of VEGF receptor tyrosine kinases VEGFR1 and VEGFR2. ${ }^{22}$ Given in combination with conventional chemotherapy, BV significantly improves the survival of patients with metastatic colorectal and lung cancer ${ }^{23,24}$ and PFS of patients with breast cancer. ${ }^{25} \mathrm{BV}$ with irinotecan has been approved by the US Food and Drug Administration (FDA) for colorectal cancer, as a first line treatment for non-small cell lung cancer in combination with carboplatin and paclitaxel, and has obtained accelerated approval for metastatic HER2-negative breast cancer patients in combination with paclitaxel. Encouraging radiographic responses and PFS have been observed in MG exposed to BV (Avastin, Genentech, CA, USA) when used in combination with irinotecan. ${ }^{26}$ In the first phase II trial published, a radiographic response rate of $63 \%$ was observed ( 1 complete response [CR] and 19 partial responses [PRs]). In addition, a 6-month PFS of $32 \%$ was obtained in GBM patients. Due to the encouraging radiographic response rate observed, the initial trial was expanded to include a total of 68 patients with recurrent MG. In the total group of 68 patients, 35 patients had a pathological diagnosis of GBM while 33 had anaplastic glioma. Among all 35 recurrent GBM patients, the 6-month PFS was $46 \%$ (95\% CI, 32\%-66\%) and the 6-month OS was $77 \%$ (95\% CI, 64\%-92\%). Twenty of the 35 patients $(57 \%$; 95\% CI, 39\%-74\%) had at least a PR. One patient developed a CNS hemorrhage and 4 patients developed thromboembolic complications (deep venous thrombosis and/or pulmonary emboli). ${ }^{27}$ Similarly, among 33 recurrent anaplastic glioma patients, the 6-month PFS was 55\% (95\% CI, 36\%-70\%) and the 6-month OS was 79\% (95\% CI, 61\%-89\%). Twenty patients $(61 \%)$ had at least a PR. Significant adverse events were infrequent and included one patient with symptomatic CNS hemorrhage and one patient who developed thrombotic thrombocytopenic purpura (TTP). The patient with CNS hemorrhage required hospitalization and high-dose dexamethasone, but made a full recovery following rehabilitative therapy. The patient who developed TTP remains on peritoneal dialysis without sign of disease progression. ${ }^{28}$

The preliminary results of a phase II, randomized, multicenter, non-comparative clinical trial of BV alone or in combination with irinotecan for GBM patients at first or second recurrence have been published. ${ }^{29}$ Eighty-five patients were randomized to the $\mathrm{BV}$ alone group and 82 to the combination of BV plus irinotecan group. Patients in the BV alone group were allowed to receive the combination of BV and irinotecan at the time of disease progression at the discretion of the investigator. Median OS were comparable at 9.2 months (95\% CI, 8.2-10.7 months) for the BV alone group and 8.7 months (95\% CI, 7.8-10.9 months) for the combination group. However, the 6-month PFS was higher in the combination group $(50.3 \%$; 95\% CI, 36.8\%-63.9\%) than in the BV-alone group (42.6\%; 95\% CI, 29.6\%-55.5\%). Grade 3 and higher toxicities were higher in the combination group, $65.8 \%$ vs $46.4 \%$ for the BV alone group, but grade 5 adverse events were more frequent in the BV-alone group $(2.4 \%$ vs $1.3 \%$ for the combination group). ${ }^{29}$

The unprecedented increase in PFS and response rate observed in MG patients when treated with the combination of $\mathrm{BV}$ and irinotecan has stimulated research with $\mathrm{BV}$ as well as other VEGF-directed or antiangiogenic therapies. Ongoing studies with agents more commonly used in MG patients are evaluating alternative BV-based regimens for recurrent MG patients. Two separate single-group studies combining BV with protracted, metronomic dosing schedules of either TMZ or etoposide are underway, as well as a study combining bortezomib, the first proteasome inhibitor, and BV. Preliminary results on these studies have not yet been published. The Radiation Therapy Oncology Group (RTOG) is randomizing recurrent GBM patients to receive BV with either protracted TMZ $\left(75 \mathrm{mg} / \mathrm{m}^{2} /\right.$ day for 21 days each month) or irinotecan every 2 weeks. An additional study combining BV plus daily erlotinib is also underway.

Potentiation of RT by the inhibition of VEGFR signaling in GBM models has been demonstrated in preliminary 
studies. ${ }^{30,31}$ Several single institutional studies are underway to evaluate the addition of BV to RT and TMZ for newly diagnosed GBM patients. The results of the interim analysis for safety and tolerability of the first 10 patients treated by the UCLA group has been published. ${ }^{32}$ The safety analysis showed that one patient experienced a presumed radiation-induced optic neuropathy. The toxicities that could be potentially related to the treatment combination were relatively high incidences of fatigue (20\% grade 3-4 post RT), myelotoxicity ( $20 \%$ and $30 \%$ grade $3-4$ during $\mathrm{RT}$ and post RT, respectively), wound breakdown (10\% and $10 \%$ grade $3-4$ during RT and post RT, respectively), and deep venous thrombosis (30\% grade $3-4$ post RT) and pulmonary embolism ( $20 \%$ grade $3-4$ post RT). They concluded that the observed toxicities were acceptable for continued enrollment toward the overall target group of 70 patients. In addition, a multi-center, randomized phase III clinical trial for newly diagnosed GBM patients is being planned.

Receptor tyrosine kinases (RTKs) inhibitors are typically small molecules that competitively block tyrosine or serine/ threonine kinase domains located intracellularly. Preliminary results of VEGF RTK inhibitors under evaluation for $\mathrm{MG}$ patients have been reported. Nine of 16 patients $(56 \%)$ treated with cediranib (AZD2171, AstraZeneca, UK), a potent, oral, pan-VEGFR, PDGFR and c-kit inhibitor, achieved a radiographic response while 3 additional patients achieved stable disease. In addition, 8 of 11 patients (73\%) were able to reduce pre-treatment corticosteroid dosing. A median PFS of 111 days and median OS of 211 days were observed in this small cohort of patients. Collaborative imaging studies revealed that decreased contrast enhancement was accompanied by significant decreases in tumor vessel size, permeability, blood volume and flow, consistent with "normalization" of tumor vessels. Of note, reversal of tumor vessel normalization was observed following drug interruption. ${ }^{33}$ The authors concluded that similar to other antiangiogenic therapies that target only tumor endothelium, monotherapy with AZD2171 may not improve overall survival, suggesting the need to combine cytotoxic therapies with AZD2171. A multi-center, randomized clinical trial is planned to evaluate cediranib versus lomustine versus the combination of cediranib plus lomustine in patients with recurrent GBM.

VEGF-TRAP (Regeneron, Inc., NY, USA), a 110 kDa soluble protein containing extracellular VEGF receptor sequences (VEGFR1 and VEGFR2) fused to a $\mathrm{IgG}$ backbone, ${ }^{34}$ acts as a soluble protein decoy VEGF receptor that binds circulating VEGF, thereby preventing it from interacting with its receptors on tumor endothelial cells. ${ }^{35,36}$ VEGF-TRAP potentates RT in prelicinical GBM xenografts. ${ }^{37} \mathrm{~A}$ phase II trial of VEGF-TRAP monotherapy for patients with TMZ-resistant recurrent GBM or anaplastic glioma at first relapse was completed by the North American Brain Tumor Consortium (NABTC). Forty-eight patients were enrolled (32 GBM and 16 anaplastic glioma). Response rates of $50 \%$ for the anaplastic glioma cohort and $30 \%$ for the GBM cohort were observed. Grade 3 adverse events included fatigue, hypertension, hand-foot syndrome, lymphopenia, thrombosis and proteinuria. One ischemic stroke and one systemic hemorrhage (grade 4 toxicities) were reported. They also observed that VEGF-TRAP induced a rapid and prolonged decrease in free levels of VEGF and PIGF, confirming the sequestration of target growth factors. ${ }^{38} \mathrm{~A}$ multi-center clinical trial incorporating VEGF-TRAP with RT and TMZ is planned for newly diagnosed GBM patients.

Vatalanib (PTK787/ZK222584; Novartis) is a novel, oral, small-molecule ATP-mimetic inhibitor of VEGFRs that has shown anti-glioma activity in preclinical studies. ${ }^{39}$ A phase I multi-institutional trial of PTK787 as monotherapy found that the agent was well tolerated with dose-limiting toxicities of deep vein thrombosis, liver enzyme elevation, insomnia, cerebral edema, fatigue, and nausea/vomiting. ${ }^{40}$ Of 31 evaluable patients, one patient presented a PR (response rate of 4\%) and 20 patients experienced disease stabilization (65\%). ${ }^{41}$ Combination of vatalanib with either TMZ or lomustine demonstrated response rate of $8 \%$ and time-to-progression of 16.1 weeks for the TMZ group and 12.1 weeks for the lomustine group. ${ }^{42}$

\section{Epidermal growth factor}

Another attractive therapeutic target is the epidermal growth factor receptor (EGFR). Approximately half of GBM tumors exhibit amplification of EGFR. In addition, EGFR is overexpressed in many MG independently of the amplification status. ${ }^{43}$ EGFRvIII, a mutant EGFR variant, is present in approximately $40 \%$ of GBM tumors, and its expression confers a negative prognosis. ${ }^{44}$ Gefinitib (ZD1839, Iressa ${ }^{\circledR}$; AstraZeneca) is a novel, oral, low-molecular weight ATP mimetic of the anilinoquinazoline family that reversibly inhibits the tyrosine kinase activity of EGFR. A phase II trial of gefinitib in first relapse GBM showed a median event-free survival of 8.1 weeks, a 6-month PFS of $17 \%$, and no radiographic responses. ${ }^{45}$ Other studies of gefitinib have failed to show survival benefits. ${ }^{46,47}$ Erlotinib (OSI-774, Tarceva ${ }^{\circledR}$; OSI Pharmaceuticals) is an orally active quinazoline derivative 
that inhibits EGFR-specific tyrosine phosphorylation and has demonstrated antitumor efficacy similar to that of gefinitib in preclinical studies. A phase I trial of erlotinib as monotherapy or in combination to $\mathrm{TMZ}$ demonstrated a response rate of $14 \%$ and a 6 -month PFS of $11 \% .{ }^{48}$ Subsequent phase II trials of erlotinib have demonstrated response rates of $6 \%$ to $25 \%$ with minimal effect on the progression rate and survival. ${ }^{49,50}$ A benefit in stratifying patients for EGFR targeted therapies has been suggested by two recent studies, demonstrating that co-expression of normal PTEN and mutant EGFRvIII, ${ }^{51}$ and combined low levels of AKT and overexpression of EGFR, ${ }^{52}$ predict radiographic responses in $\mathrm{MG}$ patients treated with erlotinib or gefitinib.

\section{Platelet derived growth factor}

Imatinib mesylate (Gleevec ${ }^{\circledR}$; Novartis, NJ, USA), a kinase inhibitor of PDGFR, c-Kit and bcr-abl, has limited anti-glioma activity when administered as monotherapy ${ }^{53}$ and in combination with RT. ${ }^{54}$ Specifically, the response rate was less than 6\% and the 6-month PFS was less than $16 \%$ in recurrent MG patients. ${ }^{55}$ In contrast, Dresemann demonstrated encouraging anti-glioma activity when imatinib was combined with hydroxyurea. ${ }^{56}$ Two subsequent phase II studies confirmed this observation. A response rate of $9 \%$ and a 6 -month PFS of $27 \%$ were observed in recurrent GBM patients ${ }^{57}$ and a response rate of $10 \%$ and a 6 -month PFS of $24 \%$ were observed in recurrent $\mathrm{AA} / \mathrm{AO}$ patients. ${ }^{58} \mathrm{~A}$ phase II, open-label, multi-centre, single-group study evaluating the efficacy of imatinib plus hydroxyurea in patients with progressive GBM that are receiving enzyme-inducing anticonvulsant drugs (EIACDs) has completed enrollment and analysis is underway.

\section{RAF-MEK-ERK}

The RAF-MEK-ERK signal transduction pathway, an important mediator of dysregulated glioma cell proliferation and angiogenesis, also offers potential therapeutic targets. In the cytoplasmic part of cell membrane, small guanine triphosphate (GTP)-binding proteins, encoded by the RAS superfamily of genes, regulate numerous cellular functions including proliferation, differentiation, cytoskeletal organization, protein trafficking, and the secretion of angiogenic factors. Mutation or amplification of upstream growth factor receptors in gliomas often result in increased RAS activity. ${ }^{59}$ Farnesylation is the rate-limiting step in RAS maturation; ${ }^{60}$ therefore, several farnesyltransferase inhibitors (FTI) have undergone clinical evaluation as RAS targeted therapy. However, FTI activity may not be specific to RAS, as many other oncoproteins also undergo farnesylation.
Tipifarnib (Zarnestra ${ }^{\circledR}$ R115777; Johnson and Johnson, NJ, USA) and lonafarnib (Sarasar ${ }^{\circledR}$, SCH66336; Schering-Plough, NJ, USA) may inactivate RAS by inhibiting farnesyltransferase. In a phase I/II study, tipifarnib demonstrated a 6-month PFS of $9 \%$ in recurrent $\mathrm{AA} / \mathrm{AO}$ and of $12 \%$ in recurrent GBM. ${ }^{61}$ Lonafarnib was evaluated in a phase I trial in combination with $\mathrm{TMZ}$ for patients with prior TMZ failure. A response rate of $27 \%$ and a 6-month PFS of $33 \%$ was observed. ${ }^{62}$ Additional molecules inhibiting components of the RAF-MEK-ERK pathway are under investigation and include AAL881 (Novartis) ${ }^{63}$ and sorafenib (Nexavar; Bayer, CT, USA).

\section{PI3K/AKT/mTOR}

PI3K, a serine/threonine kinase activated by several receptor tyrosine kinases, active RAS, or integrins, regulates several malignant phenotypes including apoptosis, cell growth, and proliferation. Poor prognosis of MG patients is associated with the activation of PI3K pathways. ${ }^{64}$ A constitutive activation of PI3K pathways is seen with PTEN loss, a common genetic feature in GBM. Activated PI3K phosphorylates several downstream effectors including AKT, another serine/threonine kinase regulating apoptosis, cell growth, and proliferation. Inhibitors of PI3K and AKT have undergone preclinical evaluation with encouraging results. ${ }^{65}$ Perifosine (Keryx Biopharmaceuticals, New York, $\mathrm{NY}$ ), an oral AKT inhibitor, administered as an oral loading dose of $600 \mathrm{mg}$ on day one followed by $100 \mathrm{mg}$ nightly thereafter is undergoing clinical evaluation in MG. Preliminary results showed a response rate of $15 \%$, but updated data are to come. ${ }^{66}$

mTOR, a serine/threonine kinase downstream from AKT, is activated not only by AKT but also by RAS pathways. Rapamycin (sirolimus; Wyeth, Madison, NJ) and its synthesized analogs, temsirolimus (CCI-779; Wyeth), everolimus (RAD001; Novartis), and AP23573 (Ariad Pharmaceuticals, Cambridge, MA, USA) have been evaluated in clinical trials of MG. Two recent phase II studies of temsirolimus in recurrent GBMs by the NABTC and the NCCTG have demonstrated modest efficacy. ${ }^{67,68}$ A radiographic response rate of 5\% and 36\%, and a 6-month PFS of $2.5 \%$ and $7.8 \%$, were observed in the NABTC and NCCTG trials, respectively. ${ }^{67,68}$ The NCCTG trial demonstrated that a predictor of radiographic response was a high level of p70s6K phosphorylation in tumor at baseline. ${ }^{68}$ A stimulation of the kinase activity of AKT, the immediate upstream effector of mTOR, has been demonstrated following the inhibition of mTOR in preclinical studies. ${ }^{69}$ PI-103, a novel inhibitor of both PI3K and mTOR, has shown promising activity in both 
in vitro and in vivo models of malignant gliomas, possibly by blocking the activated PI3K/AKT induced by the mTOR inhibition. ${ }^{70}$

\section{Protein kinase C}

High dose tamoxifen has been shown to inhibit protein kinase $\mathrm{C}$ (PKC), a serine/threonine kinase that regulates cell proliferation, invasion and angiogenesis, and to have anti-tumor activity in glioma xenografts. ${ }^{71}$ However, tamoxifen mixed results have been reported in clinical trials. ${ }^{72-78}$ Enzastaurin (LY317615; Eli-Lilly, IN, USA), an oral serine/threonine kinase inhibitor targeting PKC and AKT pathways, induces tumor cell apoptosis and suppresses proliferation and angiogenesis. ${ }^{79} \mathrm{~A}$ response rate of $29 \%$ was reported in a phase II trial of recurrent MG ${ }^{80}$ Unfortunately, a multi-centered phase III trial of enzastaurin versus lomustine in patients with recurrent GBM was discontinued at the interim analysis due to lack of benefit over the control group. ${ }^{81}$ Patients were randomized 2:1 to receive 6-week cycles of $500 \mathrm{mg}$ of enzastaurin daily (125-mg loading dose day 1) or lomustine (100-130 mg/m² on day 1). Enrollment was terminated at 266 patients after a planned interim analysis for futility. Median PFS (HR $=1.28[0.97,1.70]$ ), $\mathrm{OS}(\mathrm{HR}=1.2[0.88,1.65])$ and a 6-month PFS rate were not different between groups. Four patients discontinued enzastaurin due to drug-related serious adverse events (AE) (erysipelas, aortic thrombosis, cerebral hemorrhage, and convulsion). Eleven (7\%) patients on enzastaurin died (4 due to AEs, 1 of which was drug-related). In the lomustine group, all four $(5 \%)$ deaths were disease-related. Grade 3-4 hematological toxicities were significantly higher for lomustine ( $\mathrm{p} \leq 0.001)$. No anemia, neutropenia, or leukopenia occurred on enzastaurin, and only 1 patient had thrombocytopenia vs 21 on lomustine. There were no significant differences in grade 3-4 non-hematological toxicities between arms. The authors concluded that enzastaurin had a better toxicity profile but was not superior to lomustine in patients with recurrent GBM. ${ }^{81}$

\section{Integrins}

Integrins are cell adhesion molecules important in glioma cell invasion, migration, proliferation, survival, and angiogenesis by their interactions with multiple extracellular ligands, including vitronectin, fibronectin, laminin, fibroblast-growth factor, MMP-2, thrombospondin, fibrin, and fibrinogen. ${ }^{82-85}$ Cilengitide (EMD121974; EMD Pharmaceuticals, NC, USA), an intravenous inhibitor of $\alpha \mathrm{v} \beta 3$ and $\alpha v \beta 5$ integrin receptors demonstrated the absence of dose-limiting toxicity and a radiographic response rate of $10 \%$ in a phase I trial for MG. ${ }^{86}$ A phase II study of single-agent cilengitide randomized recurrent GBM patients to either an intermediate-low dose $(500 \mathrm{mg})$ or an intermediate-high dose $(2000 \mathrm{mg})$. No reproducible toxicities were observed in either group, and outcome trended more favorably among patients treated at the higher dose level, including a 6-month PFS of $15 \%$ vs $9.7 \%$ and a 12 -month OS of $37.5 \%$ (95\% CI $22.7 \%-54.2 \%$ ) vs $22 \%$ (95\% CI 10.6-37.6).$^{87}$ Radiographic response occurred in both groups but a higher rate was observed among patients treated at $2000 \mathrm{mg}$ (12.5\%) compared to patients treated with $500 \mathrm{mg}$ (4.8\%). Also, preliminary results of a multi-center trial of cilengitide (500 mg twice weekly) in addition to RT and TMZ for patients with newly diagnosed GBM patients recently reported a 6-month PFS of 69\% (95\% CI 55\%-83\%) and a 12 -month OS of $67 \%$ (95\% CI $42 \%-92 \%)$. Also, no added toxicity was observed when cilengitide was added to RT and TMZ. ${ }^{88}$ Additional studies with cilengitide are ongoing or planned including a trial evaluating the intratumoral pharmacodynamics and pharmacokinetics of cilengitide in recurrent GBM patients treated with cilengitide prior to a planned debulking procedure by the NABTC, a trial evaluating cilengitide dosed at $2000 \mathrm{mg}$ twice weekly in combination with RT and TMZ by the New Approaches to Brain Tumor Therapy (NABTT) and a multi-center, randomized phase III trial for newly diagnosed GBM patients.

\section{Combination therapies}

To date, the presence of multiple parallel and/or compensatory pathways and MG heterogeneity likely contributes to the limited activity observed with single agent therapy using molecularly targeted agents in MG patients. Multiple strategies are being evaluated to help overcome these factors. One such strategy is the use of tyrosine kinase inhibitors with multiple targets and a second strategy is to combine them with cytotoxic agents.

\section{Tyrosine kinase inhibitors with multiple targets}

AEE788 (Novartis), a dual inhibitor of EGFR and VEGFR-2, has shown preclinical efficacy in murine models of glioblastoma ${ }^{89}$ Vandetanib (ZD6474, Zactima ${ }^{\circledR}$; AstraZeneca), another dual inhibitor of EGFR/VEGFR-2, also demonstrated survival benefits in a murine model of intracranial glioma xenografts. ${ }^{90}$ Clinical trials of vandetanib in MG are ongoing. Sunitinib malate (Sutent ${ }^{\circledR}$, SU11248; Pfizer), an inhibitor of VEGFR-2, PDGFR, c-KIT and FMS-like 
tyrosine kinase (FLT)-3, has activity against a subcutaneous malignant glioma xenograft. ${ }^{91}$ A phase II study of sunitinib malate in $\mathrm{MG}$ is under development.

\section{Targeted therapies combined with cytotoxic agents}

Promising activity has been observed with imatinib mesylate in combination with hydroxyurea (see above). Also, a phase I trial of imatinib mesylate with temozolomide is underway. ${ }^{92}$ Gefitinib $^{93}$ and erlotinib ${ }^{48}$ have been evaluated in combination with TMZ. In an effort to enhance the sensitivity of glioma cells to RT, multiple agents are evaluated in combination with RT including gefitinib and erlotinib with or without TMZ, ${ }^{94}$ imatinib mesylate, tipifarnib,${ }^{95}$ mTOR inhibitors,${ }^{96}$ vandetanib and $\mathrm{BV}$.

\section{Locoregional therapies}

Locoregional therapies are promising approaches due to their ability to circumvent the BBB, to minimize systemic toxicity, and to concentrate therapy at the primary tumor site, which is well-recognized to be the site of tumor recurrence in most MG patients. ${ }^{97,98}$ Gliadel $^{\circledR}$, a controlled-release, biodegradable polymer releasing carmustine (BCNU), was the first approved locoregional therapy for $\mathrm{MG} .{ }^{99}$ Double-blind, randomized, placebo-controlled studies using Gliadel implantation in surgically resectable cases provided an 8-week survival benefit and a 2.3-month survival benefit in recurrent GBM and newly diagnosed $\mathrm{MG}$, respectively. ${ }^{100,101}$

Delivery of high dose radiation to the tumor bed via stereotactic radiosurgery (SRS) is another locoregional strategy. However, among newly diagnosed GBM patients, no improvement in survival was seen when SRS was added to conventional RT plus BCNU chemotherapy. ${ }^{102}$ The role of brachytherapy with ${ }^{125}$ I-beads implanted into the resection cavity is limited by a high rate of radiation necrosis requiring surgical debulking. ${ }^{103-105}$ GliaSite $^{\circledR}$, a commercially available product consisting of an aqueous solution of organically bound ${ }^{125} \mathrm{I}$ (Iotrex ${ }^{\mathrm{TM}}$ [sodium 3-((125)I)-iodo-4hydroxybenzene-sulfonate]; Cytyc Corp, Marlborough, MA) that delivers low-dose-rate radiation via a temporarily inflated balloon catheter following resection, is undergoing evaluation for newly diagnosed and recurrent MG. Modest results have been observed thus far with a 1 year survival rate of $31.1 \%$ for patient with recurrent WHO grade III and IV. ${ }^{106}$ Encouraging survival benefits have been noted in single group studies evaluating the administration of radiolabeled antitenascin monoclonal antibodies into the resection cavity of newly diagnosed and recurrent $\mathrm{MG}$ patients, with a low rate of radia- tion necrosis requiring surgical debulking. ${ }^{107,108}$ In a phase II study evaluating the administration of $100 \mathrm{mCi}$ of ${ }^{131} \mathrm{I}-\mathrm{m} 81 \mathrm{C} 6$ followed by chemotherapy to recurrent MG patients, a median OS for patients with GBM and WHO grade III tumors of 64 and 99 weeks respectively was observed. ${ }^{109}$ A multi-center phase III randomized study of 131I-labeled anti-tenascin murine monoclonal antibody (Neuradiab; Bradmer Pharmaceuticals, Inc, Toronto, ON) in combination with RT and $\mathrm{TMZ}$ versus RT and TMZ in patients with newly diagnosed GBM has just been initiated.

Convection enhanced delivery (CED) involves the gradual infusion of a therapeutic molecule over 3 to 5 days via microinfusion catheters strategically placed in the peritumoral region. The consistent positive infusion pressure gradient achieved by CED offers the possibility to overcome the increased intratumoral interstitial pressure that limits the intracranial delivery of systemically administered therapeutics for MG patients. ${ }^{110}$ In this manner, CED can potentially deliver therapeutic agents homogeneously into clinically significant volumes of distribution. ${ }^{111}$ Convection enhanced delivery of IL-13 conjugated with pseudomonas exotoxin (cintredekin besudotox, IL13-PE38QQR; NeoPharm, IL, USA) had shown encouraging results in phase I/II study for MG. ${ }^{112}$ Unfortunately, a recently completed phase III trial with this agent administered by CED failed to achieve a survival benefit compared to control patients randomized to receive carmustine wafers $\left(\right.$ Gliadel $\left.^{\circledR}\right) .{ }^{113}$ The evaluation of additional molecules or toxins via CED is underway.

\section{Conclusions and future directions}

Given the dismal prognosis associated with traditional cytotoxic agents for MG patients, there is an urgent need for more effective therapies. Thanks to intensive laboratory research conducted over the past several years, many molecular genetic and signal transduction pathway targets have been identified that are now being evaluated therapeutically. The identification of tumor phenotypes, MGMT gene methylation status, EGFRvIII, PTEN, and so on, have already demonstrated their significance (improved prognosis of patients with MGMT gene methylation treated with TMZ, ${ }^{10}$ better radiographic response to erlotinib for patients demonstrating co-expression of normal PTEN and mutant EGFRvIII $\left.{ }^{51}\right)$. However, additional information and understanding is necessary, as the inhibition of one pathway is known to induce the activation of a second pathway. In addition, novel locoregional treatments as well as strategies to improve regional delivery are being evaluated. Given the challenges posed by the heterogeneity within and across 
malignant glioma tumors, it is likely that combinations of these approaches, as well as better identification of factors predictive of response or failure to specific agents among patients, will be required as we move forward to improve the outcome of MG patients.

\section{Drug and compounds}

Temozolomide (Temodar ${ }^{\circledR}$,Temodal ${ }^{\circledR}$; Schering-Plough Corporation, NJ, USA); $\mathrm{O}^{6}$-benzylguanine; lomustine; BCNU; bevacizumab (Avastin ${ }^{\circledR}$; Genentech, CA, USA); Vatalanib (PTK787/ZK222584; Novartis); Gefinitib (ZD1839, Iressa ${ }^{\circledR}$; AstraZeneca, UK); Erlotinib (OSI774, Tarceva ${ }^{\circledR}$; OSI Pharmaceuticals); imatinib mesylate (Gleevec $^{\circledR}$; Novartis, NJ, USA); hydroxyurea; tipifarnib (Zarnestra $^{\circledR}$, R115777; Johnson and Johnson, NJ, USA); lonafarnib (Sarasar ${ }^{\circledR}$, SCH66336; Schering-Plough, NJ, USA); AAL881 (Novartis); sorafenib (Nexavar ${ }^{\circledR}$; Bayer, CT, USA); tamoxifen; enzastaurin (LY317615, Eli-Lilly, IN, USA); cilengitide (EMD121974, EMD Pharmaceuticals, NC, USA); AEE788 (Novartis); vandetanib (ZD6474, Zactima ${ }^{\circledR}$; AstraZeneca); sunitinib malate (Sutent $^{\circledR}$, SU11248; Pfizer); Gliadel ${ }^{\circledR}$; stereotactic radiosurgery; GliaSite ${ }^{\circledR}$; radiolabeled antitenascin monoclonal antibodies; convection enhanced delivery (CED); cintredekin besudotox (IL13-PE38QQR; NeoPharm, IL, USA); cediranib (AZD2171; AstraZeneca, UK); VEGF-TRAP (Regeneron, Inc., NY, USA); perifosine (Keryx Biopharmaceuticals, New York, NY, USA); rapamycin (sirolimus; Wyeth, Madison, NJ, USA); temsirolimus (CCI-779; Wyeth); everolimus (RAD001; Novartis); AP23573 (Ariad Pharmaceuticals, Cambridge, MA, USA); PI-103 (AMG706; Amgen).

\section{Disclosures}

The authors have no conflicts of interest to disclose.

\section{References}

1. Cancer Facts and Figures 2005. Surveillance Research. American Cancer Society Inc. Atlanta, USA. 2005;1-60.

2. Statistical Report: Primary Brain Tumors in the United States, 19972001. Central Brain Tumor Registry of the United States. Hinsdale, USA. 2004.

3. Kleihues P, Cavenee WK. Pathology and genetics: Tumors of the nervous system. In: World Health Classification of tumors. International Agency for Research on Cancer, Albany, USA. 2000;314.

4. Louis DN, Ohgaki H, Wiestler OD, et al. The 2007 WHO classification of tumours of the central nervous system. Acta Neuropathol (Berl). 2007;114(2):97-109.

5. Scott CB, Scarantino C, Urtasun R, et al. Validation and predictive power of Radiation Therapy Oncology Group (RTOG) recursive partitioning analysis classes for malignant glioma patients: a report using RTOG 90-06. Int J Radiat Oncol Biol Phys. 1998;40(1):51-55.
6. Wong ET, Hess KR, Gleason MJ, et al. Outcomes and prognostic factors in recurrent glioma patients enrolled onto phase II clinical trials. J Clin Oncol. 1999;17(8):2572-2578.

7. Stewart LA. Chemotherapy in adult high-grade glioma: a systematic review and meta-analysis of individual patient data from 12 randomised trials. Lancet. 2002;359(9311):1011-1018.

8. Stupp R, Mason WP, van den Bent MJ, et al. Radiotherapy plus concomitant and adjuvant temozolomide for glioblastoma. $N$ Engl J Med. 2005;352(10):987-996.

9. Gerson SL. MGMT: its role in cancer aetiology and cancer therapeutics. Nat Rev Cancer. 2004;4(4):296-307.

10. Hegi ME, Diserens AC, Gorlia T, et al. MGMT gene silencing and benefit from temozolomide in glioblastoma. $N$ Engl J Med. 2005;352(10):997-1003.

11. Friedman HS. Can O6-alkylguanine-DNA alkyltransferase depletion enhance alkylator activity in the clinic? Clin Cancer Res. 2000;6(8):2967-2968.

12. Quinn JA, Desjardins A, Weingart J, et al. Phase I trial of temozolomide plus O6-benzylguanine for patients with recurrent or progressive malignant glioma. J Clin Oncol. 2005;23(28):7178-7187.

13. Kleihues P, Cavenee WK. Pathology and Genetics Tumours of the Nervous System. IARC Press, Lyon, 2000;314.

14. Reijneveld JC, Voest EE, Taphoorn MJ. Angiogenesis in malignant primary and metastatic brain tumors. J Neurol. 2000;247:597-608.

15. Chaudhry LH, O'Donovan DG, Brenchley PE, Reid H, Roberts IS. Vascular endothelial growth factor expression correlates with tumour grade and vascularity in gliomas. Histopathology. 2001;39:409-415.

16. Ke LD, Shi YX, Im SA, Chen X, Yung WK. The relevance of cell proliferation, vascular endothelial growth factor, and basic fibroblast growth factor production to angiogenesis and tumorigenicity I human glioma cell lines. Clin Cancer Res. 2000;6:2562-2572.

17. Plate KH, Breier G, Weich HA, Risau W. Vascular endothelial growth factor is a potential tumour angiogenesis factor in human gliomas in vivo. Nature.1992;359:845-848.

18. Samoto K, Ikezaki K. Ono M, et al. Expression of vascular endothelial growth factor and its possible relation with neovascualarization in human brain tumors. Cancer Res. 1995;55:1189-1193.

19. Longo R, Sarmiento R, Fanelli M, Capaccetti B, Gattuso D, Gasparini G. Anti-angiogenic therapy: Rationale, challenges and clinical studies. Angiogenesis. 2002;5:237-256.

20. Kim KJ, Li B, Winer J, et al. Inhibition of vascular endothelial growth factor-induced angiogenesis suppresses tumour growth in vivo. Nature. 1993;362:841-844.

21. Presta LG, Chen H, O'Connor SJ, et al. Humanization of an anti-vascular endothelial growth factor monoclonal antibody for the therapy of solid tumors and other disorders. Cancer Res. 1997;57:4593-4599.

22. Ferrara N, Hillan KJ, Gerber H, Novotny W. Discovery and development of bevacizumab, an anti-VEGF antibody for treating cancer. Nature Rev Drug Discov. 2004;3:391-400.

23. Hurwitz H, Fehrenbacher L, Novotny W, et al. Bevacizumab plus Irinotecan, Fluorouracil, and Leucovorin for Metastatic Colorectal Cancer. N Engl J Med. 2004;350:2335-2342.

24. Sandler A, Gray R, Perry MC, et al. Paclitaxel carboplatin alone or with bevacizumab for non-small cell lung cancer. $N$ Engl $\mathrm{J} \mathrm{Med}$ 2006;355:2542-2550.

25. Miller K, Wang M, Gralow J, et al. Paclitaxel plus bevacizumab versus paclitaxel alone for metastatic breast cancer. $N$ Engl J Med. 2007;357(26):2666-2676.

26. Vredenburgh JJ, Desjardins A, Herndon JE, et al. Phase II trial of bevacizumab and irinotecan in recurrent malignant glioma. Clin Cancer Res. 2007;13(4):1253-1259.

27. Vredenburgh J, Desjardins A, Herndon JE, et al. Bevacizumab plus irinotecan in recurrent glioblastoma multiforme. J Clin Oncol. 2007;25:4722-4729.

28. Desjardins A, Reardon DA, Herndon JE 2nd, et al. Bevacizumab plus irinotecan in recurrent WHO grade III malignant gliomas. Clin Cancer Res. 2008;14:7068-7073. 
29. Cloughesy TF, Prados MD, Wen PY, et al. A phase II, randomized, non-comparative clinical trial of the effect of bevacizumab (BV) alone or in combination with irinotecan (CPT) on 6-month progression free survival (PFS6) in recurrent, treatment-refractory glioblastoma (GBM). J Clin Oncol. 2008;26(May 20 suppl):abstr 2010b.

30. Geng L, Donnelly E, McMahon G, et al. Inhibition of vascular endothelial growth factor receptor signaling leads to reversal of tumor resistance to radiotherapy. Cancer Res. 2001;61(6):2413-2419.

31. Kozin SV, Boucher Y, Hicklin DJ, Bohlen P, Jain RK, Suit HD. Vascular endothelial growth factor receptor-2-blocking antibody potentiates radiation-induced long-term control of human tumor xenografts. Cancer Res. 2001;61(1):39-44.

32. Lai A, Filka E, McGibbon B, Nghiemphu PL, et al. Phase II pilot study of bevacizumab in combination with temozolomide and regional radiation therapy for up-front treatment of patients with newly diagnosed glioblastoma multiforme: interim analysis of safety and tolerability. Int J Radiat Oncol Biol Phys. 2008;71(5):1372-1380.

33. Batchelor TT, Sorensen AG, di Tomaso E, et al. AZD2171, a Pan-VEGF Receptor Tyrosine Kinase Inhibitor, Normalizes Tumor Vasculature and Alleviates Edema in Glioblastoma Patients. Cancer Cell. 2007;11(1):83-95.

34. Saishin Y, Saishin YTakahasi K, et al. VEGF-TRAP ${ }_{\text {R1R2 }}$ suppresses choroidal neovascularization and VEGF-induced breakdown of the blood-retinal barrier. J Cell Physiol. 2003;195:241-248.

35. Konner J, Dupont J. Use of soluble recombinant decoy receptor vascular endothelial growth factor trap (VEGF Trap) to inhibit vascular endothelial growth factor activity. Clin Colorectal Cancer. 2004;4 Suppl 2:S81-85.

36. Holash J, Davis S, Papadopoulos N, et al. VEGF-Trap: a VEGF blocker with potent antitumor effects. Proc Natl Acad Sci US A, 2002;99(17):11393-11398.

37. Wachsberger PR, Burd R, Cardi C, et al. VEGF trap in combination with radiotherapy improves tumor control in u 87 glioblastoma. Int $J$ Radiat Oncol Biol Phys. 2007;67(5):1526-1537.

38. De Groot JF, Wen PY, Lamborn K, et al. Phase II single arm trial of aflibercept in patients with recurrent temozolomide-resistant glioblastoma: NABTC 0601. J Clin Oncol. 2008;26(May 20 suppl): abstr 2020 .

39. Goldbrunner RH, Bendszus M, Wood J, Kiderlen M, Sasaki M, Tonn JC. PTK787/ZK222584, an inhibitor of vascular endothelial growth factor receptor tyrosine kinases, decreases glioma growth and vascularization. Neurosurgery. 2004;55(2):426-432; discussion 432.

40. Yung WKA, Friedman H, Conrad C, et al. A phase I trial of single-agent PTK 787/ZK 222584 (PTK/ZK), an oral VEGFR tyrosine kinase inhibitor, in patients with recurrent glioblastoma multiforme. Proceedings of the American Society of Clinical Oncology Meeting. Chicago, USA. 2003. Abstract 395.

41. Conrad C, Friedman H, Reardon D. A Phase I/II trial of single-agent PTK787/ZK222584 (PTK/ZK), a novel, oral angiogenesis inhibitor, in patients with recurrent glioblastoma multiforme (GBM). Proceedings of the American Society of Clinical Oncology Meeting. New Orleans, USA. 2004. Abstract 1512.

42. Reardon D, Friedman H, Yung WKA, et al. A phase I/II trial of PTK787/ ZK 222584 (PTK/ZK), a novel, oral angiogenesis inhibitor, in combination with either temozolomide or lomustine for patients with recurrent glioblastoma multiforme (GBM). Proceedings of the American Society of Clinical Oncology Meeting. New Orleans, USA. 2004. Abstract 1513.

43. Ekstrand AJ, James CD, Cavenee WK, Seliger B, Pettersson RF, Collins VP. Genes for epidermal growth factor receptor, transforming growth factor alpha, and epidermal growth factor and their expression in human gliomas in vivo. Cancer Res. 1991;51(8):2164-2172.

44. Pelloski CE, Zhang L, Lin E, et al. EGFRvIII status defines distinct subtypes of glioblastoma: an analysis of 649 cases. Proceedings of the Eleventh Annual Meeting of the Society for Neuro-Oncology. Orlando, USA. 2006. Abstract PA-26.

45. Rich JN, Reardon DA, Peery T, et al. Phase II trial of gefitinib in recurrent glioblastoma. J Clin Oncol. 2004;22(1):133-142.
46. Uhm JH, Ballman KV, Giannini C, et al. Phase II study of ZD1839 in patients with newly diagnosed grade 4 astrocytoma. Proceedings of the American Society of Clinical Oncology Meeting. New Orleans, USA. 2004. Abstract 1505.

47. Lieberman FS, Cloughesy T, Fine H, et al. NABTC phase I/II trial of ZD-1839 for recurrent malignant gliomas and unresectable meningiomas. Proceedings of the American Society of Clinical Oncology Meeting. New Orleans USA. 2004. Abstract 1510.

48. Prados MD, Lamborn KR, Chang S, et al. Phase 1 study of erlotinib $\mathrm{HCl}$ alone and combined with temozolomide in patients with stable or recurrent malignant glioma. Neuro Oncol. 2006;8(1):67-78.

49. Vogelbaum MA, Peereboom D, Stevens G, Barnett G, Brewer C. Phase II trial of the EGFR tyrosine kinase inhibitor erlotinib for single agent therapy of recurrent glioblastoma mutliforme: Interim results. Proceedings of the American Society of Clinical Oncology Meeting. New Orleans, USA. 2004. Abstract 1558.

50. Raizer JJ, Abrey LE, Wen P, et al. A phase II trial of erlotinib (OSI774) in patients (pts) with recurrent malignant gliomas (MG) not on EIAEDs. Proceedings of the American Society of Clinical Oncology Meeting. New Orleans, USA. 2004. Abstract 1502.

51. Mellinghoff IK, Wang MY, Vivanco I, et al. Molecular determinants of the response of glioblastomas to EGFR kinase inhibitors. $N$ Engl J Med. 2005;353(19):2012-2024.

52. Haas-Kogan DA, Prados MD, Tihan T, et al. Epidermal growth factor receptor, protein kinase B/Akt, and glioma response to erlotinib. J Natl Cancer Inst. 2005;97(12):880-887.

53. Kilic T, Alberta JA, Zdunek PR, et al. Intracranial inhibition of plateletderived growth factor-mediated glioblastoma cell growth by an orally active kinase inhibitor of the 2-phenylaminopyrimidine class. Cancer Res. 2000;60(18):5143-5150.

54. Geng L, Shinohara ET, Kim D, et al. STI571 (Gleevec) improves tumor growth delay and survival in irradiated mouse models of glioblastoma. Int J Radiat Oncol Biol Phys. 2006;64(1):263-271.

55. Wen PY, Yung WK, Lamborn KR, et al. Phase I/II study of imatinib mesylate for recurrent malignant gliomas: North American Brain Tumor Consortium Study 99-08. Clin Cancer Res. 2006;12(16):4899-4907.

56. Dresemann G. Imatinib and hydroxyurea in pretreated progressive glioblastoma multiforme: a patient series. Ann Oncol. 2005;16(10):1702-1708.

57. Reardon DA, Egorin MJ, Quinn JA, et al. Phase II study of imatinib mesylate plus hydroxyurea in adults with recurrent glioblastoma multiforme. J Clin Oncol. 2005;23(36):9359-9368.

58. Desjardins A, Quinn JA, Vredenburgh JJ, et al. Phase II study of imatinib mesylate and hydroxyurea for recurrent grade III malignant gliomas. J Neurooncol. 2007;83(1):53-60.

59. Knobbe CB, Reifenberger J, Reifenberger G. Mutation analysis of the Ras pathway genes NRAS, HRAS, KRAS and BRAF in glioblastomas. Acta Neuropathol (Berl). 2004;108:467-470.

60. Shaw RJ, Cantley LC. Ras, PI(3)K and mTOR signalling controls tumour cell growth. Nature. 2006;441:424-430.

61. Cloughesy TF, Wen PY, Robins HI, et al. Phase II trial of tipifarnib in patients with recurrent malignant glioma either receiving or not receiving enzyme-inducing antiepileptic drugs: a North American Brain Tumor Consortium Study. J Clin Oncol. 2006;24(22):3651-3656.

62. Gilbert MR, Gaupp P, Liu V, et al. A phase I study of temozolomide (TMZ) and the farnesyltransferase inhibitor (FTI), lonafarnib (Sarazar, SCH66336) in recurrent glioblastoma. Proceedings of the American Society of Clinical Oncology Meeting. Atlanta, USA. 2006. Abstract 1556

63. Sathornsumetee S, Hjelmeland AB, Keir ST, et al. AAL881, a novel small molecule inhibitor of RAF and vascular endothelial growth factor receptor activities, blocks the growth of malignant glioma. Cancer Res. 2006;66(17):8722-8730.

64. Chakravarti A, Zhai G, Suzuki Y, et al. The prognostic significance of phosphatidylinositol 3-kinase pathway activation in human gliomas. J Clin Oncol. 2004;22:1926-1933. 
65. Momota H, Nerio E, Holland EC. Perifosine inhibits multiple signaling pathways in glial progenitors and cooperates with temozolomide to arrest cell proliferation in gliomas in vivo. Cancer Res. 2005;65:7429-7435.

66. Lassman A, Holland E, DeAngelis L, Panageas K, Abrey L. Clinical and molecular-metabolic phase II trial of perifosine for recurrent/progressive malignant glioma. Neuro Oncol. 2007;9(4):467-602, Abstract MA-15.

67. Chang SM, Wen P, Cloughesy T, et al. Phase II study of CCI-779 in patients with recurrent glioblastoma multiforme. Invest New Drugs. 2005;23:357-361

68. Galanis E, Buckner JC, Maurer MJ, et al. Phase II trial of temsirolimus (CCI-779) in recurrent glioblastoma multiforme: a North Central Cancer Treatment Group Study. J Clin Oncol. 2005;23:5294-5304.

69. Sawyers CL. Will kinase inhibitors have a dark side? N Engl J Med. 2006;355:313-315.

70. Fan QW, Knight ZA, Goldenberg DD, et al. A dual PI3 kinase/ mTOR inhibitor reveals emergent efficacy in glioma. Cancer Cell. 2006;9:341-349.

71. Hui AM, Zhang W, Chen W, et al. Agents with selective estrogen receptor (ER) modulator activity induce apoptosis in vitro and in vivo in ER-negative glioma cells. Cancer Res. 2004;64(24):9115-9123.

72. Brandes AA, Ermani M, Turazzi S, et al. Procarbazine and high-dose tamoxifen as a second-line regimen in recurrent high-grade gliomas: a phase II study. J Clin Oncol. 1997;17(2):645-650.

73. Spence AM, Peterson RA, Scharnhorst JD, Silbergeld DL, Rostomily RC. Phase II study of concurrent continuous Temozolomide (TMZ) and Tamoxifen (TMX) for recurrent malignant astrocytic gliomas. J Neurooncol. 2004:70(1):91-95.

74. Robins HI, Won M, Seiferheld WF, et al. Phase 2 trial of radiation plus high-dose tamoxifen for glioblastoma multiforme: RTOG protocol BR-0021. Neuro Oncol. 2006;8(1):47-52.

75. Tang P, Roldan G, Brasher PM, et al. A phase II study of carboplatin and chronic high-dose tamoxifen in patients with recurrent malignant glioma. J Neuro Oncol. 2006;78(3), 311-316.

76. Puchner MJ, Herrmann HD, Berger J, Cristante L. Surgery, tamoxifen, carboplatin, and radiotherapy in the treatment of newly diagnosed glioblastoma patients. J Neurooncol. 2000;49(2):147-155.

77. Chang SM, Barker FG 2nd, Huhn SL, et al. High dose oral tamoxifen and subcutaneous interferon alpha-2a for recurrent glioma. J Neurooncol. 1998;37(2):169-176.

78. Couldwell WT, Weiss MH, DeGiorgio CM, et al. Clinical and radiographic response in a minority of patients with recurrent malignant gliomas treated with high-dose tamoxifen. Neurosurgery. 1993;32(3):485-489; discussion 489-490.

79. Graff JR, McNulty AM, Hanna KR, et al. The protein kinase Cbeta-selective inhibitor, Enzastaurin (LY317615.HCl), suppresses signaling through the AKT pathway, induces apoptosis, and suppresses growth of human colon cancer and glioblastoma xenografts. Cancer Res. 2005;65(16):7462-7469.

80. Fine HA, Kim L, Royce C, et al. Results from phase II trial of enzastaurin (LY317615) in patients with recurrent high grade gliomas Proceedings of the American Society of Clinical Oncology Meeting. Orlando, USA. 2004. Abstract 1504.

81. Fine HA, Puduvalli VK, Chamberlain MC, et al. Enzastaurin (ENZ) versus lomustine (CCNU) in the treatment of recurrent, intracranial glioblastoma multiforme (GBM): A phase III study. J Clin Oncol. 2008;26(May 20 suppl):abstr 2005.

82. Bello L, Francolini M, Marthyn P, Zhang J, Carroll RS, Nikas DC, et al. Alpha(v)beta3 and alpha(v)beta5integrin expression in glioma periphery. Neurosurgery 2001;49(2):380-389(discussion 390).

83. Gingras MC, Roussel E, Bruner JM, Branch CD, Moser RP. Comparison of cell adhesion molecule expression between glioblastoma multiforme and autologous normal brain tissue. J Neuroimmunol. 1995;57(1-2):143-153.

84. Gladson CL. Expression of integrin alpha v beta 3 in small blood vessels of glioblastoma tumors. J Neuropathol Exp Neurol. 1996;55(11):1143-1149.
85. Tucker GC: Integrins: molecular targets in cancer therapy. Curr Oncol Rep. 2006;8(2):96-103.

86. Nabors LB, Mikkelsen T, Rosenfeld SS, Hochberg F, Akella NS, Fisher JD, et al. Phase I and correlative biology study of cilengitide in patients with recurrent malignant glioma. J Clin Oncol. 2007;25(13):1651-1657.

87. Reardon DA, Fink K, Nabors LB, Cloughesy T, Plotkin S, Schiff D, et al. Phase IIa trial of cilengitide (EMD121974) single-agent therapy in patients (pts) with recurrent glioblastoma (GBM): EMD 121974- 009. In 43rd Annual Meeting of American Society of Clinical Oncology. Edited by Grunberg SM. Chicago, IL: Lisa Greaves;2007:75s.

88. Stupp R, Goldbrunner R, Neyns B, et al. Phase I/IIa trial of cilengitide (EMD121974) and temozolomide with concomitant radiotherapy, followed by temozolomide and cilengitide maintenance therapy in patients (pts) with newly diagnosed glioblastoma (GBM). J Clin Oncol, 2007 ASCO Annual Meeting Proceedings 2007;25(Pt 1):No. 18S (June 20 Supplement)

89. Goudar RK, Shi Q, Hjelmeland MD, et al. Combination therapy of inhibitors of epidermal growth factor receptor/vascular endothelial growth factor receptor 2 (AEE788) and the mammalian target of rapamycin (RAD001) offers improved glioblastoma tumor growth inhibition. Mol Cancer Ther. 2005;4(1):101-112.

90. Rich JN, Sathornsumetee S, Keir ST, et al. ZD6474, a novel tyrosine kinase inhibitor of vascular endothelial growth factor receptor and epidermal growth factor receptor, inhibits tumor growth of multiple nervous system tumors. Clin Cancer Res. 2005;11(22):8145-8157.

91. Schueneman AJ, Himmelfarb E, Geng L, et al. SU11248 maintenance therapy prevents tumor regrowth after fractionated irradiation of murine tumor models. Cancer Res. 2003;63(14):4009-4016.

92. Sathornsumetee S, Reardon DA, Quinn JA, et al. An update on phase I study of dose-escalating imatinib mesylate plus standard-dosed temozolomide for the treatment of patients with malignant glioma. Proceedings of the American Society of Clinical Oncology Meeting. Atlanta, USA. 2006. Abstract 1560.

93. Prados M, Yung W, Wen P, et al. Phase I study of ZD1839 plus temozolomide in patients with malignant glioma. A study of the North American Brain Tumor Consortium. Proceedings of the American Society of Clinical Oncology Meeting. New Orleans, USA. 2004. Abstract 1504.

94. Krishnan S, Brown PD, Ballman KV, et al. Phase I trial of erlotinib with radiation therapy in patients with glioblastoma multiforme: results of North Central Cancer Treatment Group protocol N0177. Int J Radiat Oncol Biol Phys. 2006;65(4):1192-1199.

95. Delmas C, Heliez C, Cohen-Jonathan E, et al. Farnesyltransferase inhibitor, R115777, reverses the resistance of human glioma cell lines to ionizing radiation. Int J Cancer. 2002;100(1):43-48.

96. Eshleman JS, Carlson BL, Mladek AC, Kastner BD, Shide KL, Sarkaria JN. Inhibition of the mammalian target of rapamycin sensitizes U87 xenografts to fractionated radiation therapy. Cancer Res. 2002;62(24);7291-7297.

97. Gaspar LE, Fisher BJ, Macdonald DR, et al. Supratentorial malignant glioma: patterns of recurrence and implications for external beam local treatment. Int J Radiat Oncol Biol Phys, 1992;24(1):55-57.

98. Hochberg FH, Pruitt A. Assumptions in the radiotherapy of glioblastoma. Neurology, 1980;30(9):907-911.

99. Brem H, Tamargo RJ, Olivi A, et al. Biodegradable polymers for controlled delivery of chemotherapy with and without radiation therapy in the monkey brain. J Neurosurg. 1994;80(2);283-290.

100. Brem H, Piantadosi S, Burger PC, et al. Placebo-controlled trial of safety and efficacy of intraoperative controlled delivery by biodegradable polymers of chemotherapy for recurrent gliomas. The Polymer-brain Tumor Treatment Group. Lancet. 1995;345(8956);1008-1012.

101. Westphal M, Hilt DC, Bortey E, et al. A phase 3 trial of local chemotherapy with biodegradable carmustine (BCNU) wafers (Gliadel wafers) in patients with primary malignant glioma. Neuro Oncol. 2003;5(2):79-88. 
102. Souhami L, Seiferheld W, Brachman D, et al. Randomized comparison of stereotactic radiosurgery followed by conventional radiotherapy with carmustine to conventional radiotherapy with carmustine for patients with glioblastoma multiforme: report of Radiation Therapy Oncology Group 93-05 protocol. Int J Radiat Oncol Biol Phys. 2004;60(3):853-860.

103. Shrieve DC, Alexander E 3rd, Wen PY, et al. Comparison of stereotactic radiosurgery and brachytherapy in the treatment of recurrent glioblastoma multiforme. Neurosurgery. 1995;36(2):275-282; discussion 282-274.

104. Selker RG, Shapiro WR, Burger P, et al. The Brain Tumor Cooperative Group NIH Trial 87-01: a randomized comparison of surgery, external radiotherapy, and carmustine versus surgery, interstitial radiotherapy boost, external radiation therapy, and carmustine. Neurosurgery. 2002;51(2):343-355; discussion 355-347.

105. Laperriere NJ, Leung PM, McKenzie S, et al. Randomized study of brachytherapy in the initial management of patients with malignant astrocytoma. Int J Radiat Oncol Biol Phys. 1998;41(5):1005-1011.

106. Gabayan AJ, Green SB, Sanan A, et al. GliaSite brachytherapy for treatment of recurrent malignant gliomas: a retrospective multi-institutional analysis. Neurosurgery. 2006;58(4):701-709; discussion 701-709.

107. Cokgor I, Akabani G, Kuan CT, et al. Phase I trial results of iodine131-labeled antitenascin monoclonal antibody 81C6 treatment of patients with newly diagnosed malignant gliomas. $J$ Clin Oncol. 2000;18(22):3862-3872.
108. Reardon DA, Akabani G, Coleman RE, et al. Phase II trial of murine (131)I-labeled antitenascin monoclonal antibody 81C6 administered into surgically created resection cavities of patients with newly diagnosed malignant gliomas. J Clin Oncol. 2002;20(5):1389-1397.

109. Reardon DA, Akabani G, Coleman RE, et al. Salvage radioimmunotherapy with murine iodine-131-labeled antitenascin monoclonal antibody 81C6 for patients with recurrent primary and metastatic malignant brain tumors: phase II study results. J Clin Oncol. 2006;24(1):115-122.

110. Jain RK. Delivery of molecular and cellular medicine to solid tumors. Adv Drug Deliv Rev. 2001;46(1-3):149-168.

111. Bobo RH, Laske DW, Akbasak A, Morrison PF, Dedrick RL, Oldfield EH. Convection-enhanced delivery of macromolecules in the brain. Proc Natl Acad Sci U S A. 1994;91(6):2076-2080.

112. Prados M, Kunwar S, Lang FF, et al. Final results of phase I/II studies of IL 13-PE38QQR administered intratumorally (IT) and/or peritumorally (PT) via convection-enhanced delivery (CED) in patients undergoing tumor resection for recurrent malignant glioma. Proceedings of the American Society of Clinical Oncology Meeting. Orlando, USA. 2005. Abstract 1506.

113. Kunwar S, Westphal M, Medhorn M, et al. Results from PRECISE: a randomized phase 3 study in patients with first recurrent glioblastoma multiformed (GBM) comparing cintredekin besudotox (CB) administered via convection-enhanced delivery (CED) with Gliadel wafers (GW). Neuro Oncol. 2007;9(4):467-602 Abstract MA-61. 
